Adrian Wettstein

\title{
Operation »Barbarossa« und Stadtkampf
}

\author{
Einleitung
}

Am 22. Juni 1941 eröffnete die deutsche Wehrmacht mit der Operation »Barbarossa« ihren ersten wirklich als Blitzkrieg geplanten Feldzug ${ }^{1}$. Ebenso allerdings handelte es sich bei diesem Feldzug gegen die UdSSR um einen Vernichtungskrieg. Die jüngere Forschung, und hier insbesondere der entsprechende Band der vom Militärgeschichtlichen Forschungsamt (MGFA) herausgegebenen Reihe »Das Deutsche Reich und der Zweite Weltkrieg « ${ }^{2}$, haben aufgezeigt, wie sehr diese beiden Konzepte divergierten und zudem von inneren Widersprüchen durchsetzt waren. Zusammen mit einer notorischen Fehleinschätzung der eigenen und der sowjetischen Militärkapazitäten, einer völlig unzureichenden Logistik und der Uneinigkeit der militärischen Spitzen des Deutschen Reiches über die Operationsführung, waren dies die Gründe für das Scheitern der Operation »Barbarossa « vor den Toren Moskaus.

Dieser Beitrag widmet sich einem in diesem Kontext bis heute kaum untersuchten Thema ${ }^{3}$, dass mitunter als weiterer Grund für das Scheitern der Operation Barbarossa bezeichnet werden darf: Dem Stadtkampf. In den spezifischen verkehrsgeografischen Verhältnissen Russlands und unter Bezugnahme auf die Blitzkriegstrategie der Wehrmacht musste die Eroberung von Städten zu einem militärischen Problem werden, nicht zuletzt auch wegen des oben bereits angesprochenen Ziel-

1 Zum problematischen Begriff des Blitzkriegs: Karl-Heinz Frieser, Blitzkrieg-Legende. Der Westfeldzug 1940, München 1995, S. 5-14; Uwe Bitzel, Die Konzeption des Blitzkrieges bei der deutschen Wehrmacht, Frankfurt a.M. 1991, S. 204-215.

2 Der Angriff auf die Sowjetunion. Hrsg. vom MGFA, Stuttgart 1983 (= Das Deutsche Reich und der Zweite Weltkrieg, 4).

3 Dies gilt allgemein für das, was im Folgenden unter dem Begriff »Stadtkampf « subsummiert wird. Und das obwohl das Thema verschiedene Forschungsgebiete streift, die in letzter Zeit intensive Behandlung erfuhren, etwa die Forschungen zum Totalen Krieg: An der Schwelle zum Totalen Krieg. Die militärische Debatte über den Krieg der Zukunft 1919-1939. Hrsg. von Stig Förster, Paderborn 2002 (= Krieg in der Geschichte, 13) oder zur Urbanisierung. Vgl. hierzu: Urban History, 29 (2002), 3, S. 460-508. Der dortige Stichwortindex deutet auf eine Vernachlässigung militärhistorischer Fragestellungen hin. Die Sammelbände, die in der Folge zweier Kongresse zum Thema Stadt und Krieg entstanden sind, zeigen dies ebenfalls deutlich. Bereits die Samples der gewählten Beispiele deuten daraufhin, dass der Kampf in Städten auf diesen Kongressen kein Thema war. Einzig der Luftkrieg wurde auch unter operativen Aspekten diskutiert. Städte im 2. Weltkrieg. Ein internationaler Vergleich. Hrsg. von Marlene P. Hiller, Eberhard Jäckel und Jürgen Rohwer, Essen 1991, und: Villes en guerre 1914-1945. Ed. par Philippe Chassaigne et Jean-Marc Largeaud, Paris 2004. Die einzige Monographie, die sich näher mit dem Kampf in Städten auseinandersetzt, kommt aus der militärgeografischen Forschung. Sie bietet einen reichen Fundus an Beispielen und methodischen Anmerkungen, genügt aber kritisch-historischen Anforderungen nicht überall. Gregory J. Ashworth, War and the City, London 1991. 
konflikts. Diese Überlegungen sollen am Fallbeispiel der Schlacht um Dnepropetrovsk ${ }^{4}$ konkretisiert werden.

Doch was bedeutet Stadtkampf eigentlich? Für das im Folgenden zu beschreibende Phänomen verwende ich mit dem Begriff "Stadtkampf « bewusst einen Neologismus, um der Gefahr der unreflektierten Übernahme zeitgenössischer Quellenbegriffe oder moderner militärischer Konzepte zu entgehen ${ }^{5}$. Es ist aber auch der Versuch, entgegen den gängigen militärischen Vorstellungen des Zweiten Weltkrieges, dieser Art des Gefechts eine - im Gegensatz zum Ortskampf - eigene Qualität zuzusprechen. Deshalb sollen an dieser Stelle kurz die zur Zeit des Zweiten Weltkrieges verwendeten deutschen Begriffe "Straßenkampf «, »Häuserkampf« und "Ortskampf « sowohl gegeneinander als auch zum Stadtkampf abgegrenzt werden.

Der am schwierigsten einzuordnende Terminus ist jener des "Straßenkampfes«. Er taucht wohl in den Quellen auf, ohne aber - wie die Begriffe »Ortskampf « und "Häuserkampf « - durch militärische Vorschriften definiert worden zu sein. Dies hängt wohl nicht zuletzt damit zusammen, dass er nie als rein militärischer Begriff existierte. Bereits früh wurde er auch im Zusammenhang mit Aufständen, Unruhen und Revolutionen verwendet, auch von ziviler Seite. Im Gegensatz zu den anderen drei findet er sich in jedem besseren Wörterbuch, wo aber seine Definition vage bleibt $^{6}$. Die Historiografie ist dieser vagen Anwendung gefolgt, wahrscheinlich in einer wenig reflektierten Übernahme aus den Quellen. Allerdings bleibt festzuhalten, dass der Begriff in den Quellen für Kämpfe in Städten auftritt, ohne aber einen genauen Charakter dieser Kämpfe zu umschreiben?.

Unter »Häuserkampf « verstand man im deutschen Militär die taktische Konzeption des Kampfes in einzelnen und um einzelne Gebäude. Der Häuserkampf ist Aufgabe kleinerer Einheiten, z.B. der Schützengruppen. Er bildet damit eine Voraussetzung für den Kampf in größeren, überbauten Gebieten, seien es Ortschaften oder Städte, ist aber keinesfalls mit diesem gleichzusetzen ${ }^{8}$.

Der »Ortskampf« ist ebenfalls ein militärisch-funktioneller Begriff. Er steht für den Kampf um kleinere Siedlungen, oftmals die herkömmliche Ansammlung einiger

4 Zur Eroberung von Dnepropetrovsk gibt es in der Literatur nur wenige Hinweise, da sie im Schatten der gleichzeitigen Umfassungsoperation um Kiev geschieht. Einen knappen Überblick gibt: Dieter Hoffmann, Die Magdeburger Division. Zur Geschichte der 13. Infanterie- und 13. Panzer-Division 1939-1945, Hamburg 2001, S. 132-137; angesprochen wird die Schlacht auch in: Der Angriff auf die Sowjetunion (wie Anm. 2), S. 512 (Beitrag Klink).

5 Zur Problematik der Sprache in der Militär- und insbesondere in der Operationsgeschichte: Bernd Wegner, Wozu Operationsgeschichte? In: Was ist Militärgeschichte? Hrsg. von Thomas Kühne und Benjamin Ziemann, Paderborn 2000, S. 105-113, hier: S. 111 f.

6 Vgl. z.B. Gerhard Wahrig, Deutsches Wörterbuch, Zürich 1988, S. 1238: »Kampf in den Straßen, zum Beispiel bei Revolutionen«.

7 Vgl. beispielsweise das bei Hanns von Krannhals, Der Warschauer Aufstand 1944, Frankfurt a.M. 1962 abgedruckte Quellenmaterial zum Warschauer Aufstand oder den Erfahrungsbericht eines an der Eroberung von Warschau 1939 beteiligten Pionierbataillons: In 5, Taktische Gesichtspunkte für die Durchführung des Straßenkampfes. Abschrift aus dem KTB (Heft 2) des Gebirgspionierbataillon 85 vom 11.9.-10.1.1939, Nr. 6092/41, Bundesarchiv-Militärarchiv (BA-MA), RH 12-5/311.

B Es finden sich in den Vorschriften keine direkten Hinweise für diese Definition, aber Ausbildungsunterlagen legen diese Interpretation nahe. Vgl. hierzu beispielsweise: Pionierlehrbataillon 4, Worauf kommt es an? Häuserkampf. Undatiert, vermutlich 1944, BA-MA, RH 17/229, Bl. 28. 
Bauernhäuser mit einem Kern aus mehreren festen Gebäuden. Die Heeresdienstvorschrift (H.Dv.) 300: Truppenführung9 ${ }^{9}$, das grundlegende taktische Reglement der deutschen Wehrmacht ${ }^{10}$, liefert selbst keine Definition des Ortskampfes, sondern beschreibt das Ortsgefecht folgendermaßen:

"Das Ringen um Ortschaften ist im Gefecht häufig und kann dem Gefecht in dicht besiedelten Gebieten das Gepräge geben. Die Bedeutung von Ortschaften in einem Gefecht richtet sich nach ihrer Lage im Gelände sowie nach ihrer Bauart und Größe. Zusammenhängenden Gebäudegruppen, wie ausgedehnten Industrie- und Bergwerksanlagen, kommt gleiche Bedeutung wie Ortschaften zu. Auch Städte und Großstädte können zum Kampfgebiet werden ${ }^{11}$.«

Als Ortskampf werden also alle Arten von Gefechten in bebauten Zonen, von kleineren Gebäudegruppen über Ortschaften bis hin zu Großstädten verstanden. Eine prinzipielle Unterscheidung zwischen Ortschaften und Städten wurde dabei nicht gemacht.

Diesen Begriffen stelle ich nun den Begriff »Stadtkampf« gegenüber, der, so wie ich ihn in diesem Beitrag verwende, die folgenden vier Definitionsmerkmale umfasst:

1. Die Größe der bebauten Zone: Gerade im Gegensatz zum Ortskampf findet der Stadtkampf in einer großräumig bebauten Zone statt. Hierbei geht es nicht nur um die quantitativen (mehr Truppen), sondern auch um qualitative Unterschiede (wechselnde Bebauungsstile, operative und strategische Ansätze).

2. Kampfhandlungen in der Stadt: Dieses Kriterium mag auf Anhieb banal klingen, hat aber die Intention, Belagerungen oder die oftmals aus historischer Perspektive als Schlacht um eine Stadt bezeichneten Operationen ${ }^{12}$, bei denen aber eben nur um und nicht in der Stadt gekämpft wird, auszuschließen.

3. Umfang der eingesetzten Truppen: Sie ergibt sich zumeist aus der Größe und Wichtigkeit der bebauten Zone ${ }^{13}$. Damit sollen Scharmützel, die sich wahrscheinlich zu jeder Zeit auch in Städten abspielten, ausgeschlossen werden.

4. Die Dauer der Kampfhandlungen: Sie muss mehrere Tage betragen. Damit sollen wie bei der Truppenstärke einerseits kleinere Scharmützel ausgeschlossen werden, andererseits die handstreichartige Einnahme von Städten.

Diese Definition dient als Hilfe, sollte aber keineswegs als abgeschlossen betrachtet werden. Die Grenzen dieser Definition bilden die exakten Kriterien der einzelnen Definitionsmerkmale. Wenn wir diese Definition auf das für diesen Beitrag verwendete Fallbeispiel Dnepropetrovsk anwenden, können wir folgendes festhalten: Die Größe der bebauten Zone ist in diesem Fall klar, da die Stadt Dnepropetrovsk mit 500000 Einwohnern schon als Großstadt gelten konnte. Es kam zu Kampfhand-

9 Truppenführung (T.F.), 2 Bde, Berlin 1936 (= H.Dv. 300). Für diese Arbeit wurde nur der erste Band verwendet, weshalb auf die jeweilige Erwähnung der Bandnummer verzichtet. wurde.

10 Zur Einordnung der H.Dv. 300 als Dokument der taktischen Doktrin der Wehrmacht: James Corum, The Roots of Blitzkrieg. Hans von Seeckt and the German Military.Reform, Lawrence, KS 1992, S. 199; Martin van Creveld, Fighting Power. German and U.S. Army Performance, 1939-1945, London 1983, S. 28-30.

11 H.Dv. 300 (wie Anm. 9), S. 225. Hervorhebung im Original.

12 Etwa die Schlacht um Leningrad, Schlacht um Verdun, Ypern-Schlachten usw.

13 Dies gilt nicht für die Militärgeschichte nach dem Zweiten Weltkrieg, da die Truppenzahlen allgemein sinken und viele Kämpfe in Städten im Zusammenhang mit Guerillaaktionen stehen. 
lungen in der Stadt, vor allem aber in den Vororten auf dem nordöstlichen Ufer, etwa in der Arbeitersiedlung Kamenka. Auch die sowjetischen Angriffe zur Zerschlagung des Brückenkopfes wurden in der Stadt oder am Stadtrand abgewehrt ${ }^{14}$. Die eingesetzten Truppen schließlich umfassten allein auf deutscher Seite vier Divisionen, Armee- und Korpstruppen sowie die Artillerie von weiteren Divisionen. Auf sowjetischer Seite wurden Teile von mindestens sieben Divisionen eingesetzt. Hinsichtlich der Dauer lässt sich sagen, dass vom Angriff auf die Stadt am 24. August 1941 bis zum Freikämpfen des Vorortes Kamenka mindestens zwei Wochen vergingen. Inwiefern sich die weiteren Kämpfe noch im Stadtgebiet abspielten, ist Definitionsfrage. In diesem Sinne kann hier festgehalten werden, dass es sich bei der Schlacht um Dnepropetrovsk im Sinne der oben gemachten Definition um einen Stadtkampf handelte.

Da die folgenden Aussagen zu der Schlacht um Dnepropetrovsk weitgehend auf Kriegstagebüchern (KTB) der Kommandobehörden im Feld basieren, seien mir hier noch einige Gedanken zum besonderen Charakter dieser Quellen erlaubt. Denn obwohl diese Quellen regelmäßig verwendet werden, fehlt bis heute eine tiefer gehende methodische Auseinandersetzung mit dieser Quellengattung ${ }^{15}$.

Wertvolle Hinweise zur Bewertung der Quelle liefern die von der Kriegswissenschaftlichen Abteilung des Generalstabes des Heeres herausgegebenen "Bestimmungen für die Führung von Kriegstagebüchern und Tätigkeitsberichten « ${ }^{16}$. Im ersten Punkt der Bestimmungen werden folgende drei Zwecke des Kriegstagebuches bzw. des Tätigkeitsberichtes genannt: Sammeln von Erfahrungen für Ausbildung und Führung, Tätigkeitsnachweis von Kommandobehörden sowie »unentbehrliche Unterlage für die Geschichtsschreibung ${ }^{17}$. Trotz der im sechsten Punkt gemachten, klaren und deutlichen Bestimmung, dass auch »der ungünstige Verlauf einer Kampfhandlung [...] wahrheitsgetreu dargestellt werden « müsse ${ }^{18}$, ist bereits hier erkenntlich, dass es zu einem Interessenkonflikt beim Schreibenden kommen musste. Auch wenn dem Tagebuchführer die ausgeprägte militärische Professionalität zusammen mit der klaren Bestimmung gebot, Fehler und Niederlagen möglichst präzise niederzuschreiben, so wusste er auf der anderen Seite dennoch genau, dass es sich hier auch um einen Rechenschaftsbericht handelte, der über die spätere Bewertung der Führer und des Verbandes bestimmte, unter Umständen die eigene militärische Karriere beeinflusste. So sind denn auch in den von mir untersuchten Teilen der Tagebücher klare Worte hinsichtlich ungünstig verlaufener Gefechte

14 Gefechtsbericht des beim I./S.R. 66 unterstellten 1. Zuges - ohne 2 Gruppen - der 3./ Pz.Pi. 4 beim Angriff auf den Nordteil Dnepropetrovsks sowie Bildung und Sicherung eines engen Brückenkopfes am 25.8.1941, BA-MA, RH 27-13/46 (Gefechtsbericht 1./3./Pz.Pi. 4).

15 Es ist klar, dass dieser Aufsatz die Lücke nicht schließen, wohl aber doch einige Anregungen zu einer tieferen Auseinandersetzung mit der Quellenart bieten kann.

16 Oberkommando des Heeres (OKH)/Kriegswissenschaftliche Abteilung Generalstab des Heeres (Gen.St.d.H.), Bestimmung über die Führung von Kriegstagebüchern und Tätigkeitsberichten, Berlin 1940 (In: 13. Pz.Div./Abt. Ia, KTB.Nr. 5, BA-MA, 27-13/12). Diese Bestimmungen fanden sich aber auch in anderen Divisionstagebüchern. Für das Jahr 1941 ließen sich keine neueren oder überarbeiteten Versionen feststellen, sodass davon ausgegangen werden kann, dass die hier genannten Bestimmungen während der Operation "Barbarossa" aktuell waren.

Ebd., S. 1.

18 Ebd. 
äußerst selten, während gleichzeitig Erfolge anderer Einheiten eingeschränkt wer$\operatorname{den}^{19}$.

Mit der Führung des KTBs beauftragt werden sollten »geeignete Offiziere, die das erforderliche taktische Verständnis besitzen [...] Sie müssen laufend insbesondere über Feindmeldungen, Lagebeurteilungen, Entschlüsse und Maßnahmen unterrichtet werden. Alle außerdem erforderlichen Unterlagen sind ihnen zugänglich zu machen ${ }^{20}$." Entscheidend war also das taktische Verständnis, was der hohen Bewertung des operativen Denkens im deutschen Militär entsprach. Allerdings musste auch der beste Stabsoffizier vor der schieren Menge an Informationen, die in einer größeren Schlacht auf ihn einströmten, kapitulieren - insbesondere wenn das Kriegstagebuch täglich geführt werden sollte. In Punkt 6 folgen weitere Forderungen, denen die untersuchten KTBs aber nur noch in Einzelfällen gerecht werden können. Während die einen KTBs der Forderung nach detaillierten Zeitangaben nachkommen, folgen andere mehr dem Wunsch, dass »die Grundlagen für das Handeln der Führer klar erkennbar sein « müsse ${ }^{21}$. Vieles aber blieb Stückwerk, was unter dem Druck der Ereignisse nicht verwundern kann. Auch die verlangten umfassenden Anlagen wurden nur teilweise beigefügt. So bleibt unklar, nach welchen Kriterien Gefechts- und Erfahrungsberichte verfasst wurden, die teilweise über längere Zeiträume fehlen oder mit monatelanger Verspätung entstanden sind. Aus diesem Grunde sollte insbesondere mit statistischen Aussagen vorsichtig verfahren werden. Auch die beigefügten Bilder und Skizzen sind kaum mehr in einen Kontext zu setzen, da eine genauere Beschriftung oder Erläuterung fehlt ${ }^{22}$.

Trotz der einheitlichen Bestimmungen weisen sowohl die Tagebücher als auch die Tätigkeitsberichte starke Unterschiede auf, die sich wahrscheinlich aus den individuellen Ausprägungen des Schreibenden ergeben haben. So ist das Tagebuch der 60. Infanteriedivision (mot.) für den hier untersuchten Zeitraum als eines der wenigen weitgehend handschriftlich verfasst ${ }^{23}$, während die anderen Tagebücher mit Schreibmaschine geschrieben wurden. Das KTB der 13. Panzerdivision ist in einem eher erzählenden Stil gehalten ${ }^{24}$, die meisten anderen KTBs geben eine mehr oder minder genaue Chronologie wieder, die sich bisweilen im Aufzählen von Befehlen mit dem Vermerk auf die entsprechenden Anlagen erschöpft. Noch größere Differenzen bestehen bei den Tätigkeitsberichten: Der Divisionsarzt der 60. Infanteriedivision (mot.) schreibt einen umfassenden Bericht ${ }^{25}$, in dem neben Zahlen, Befehlen und Verschiebungen auch eine ganze Menge an Erfahrungen weitergegeben wird. Der Arzt der Panzergruppe 1 bleibt dagegen militärisch kurz und erzählt

19 Dies schlägt sich innerhalb des hier gewählten Fallbeispieles so nieder, dass die unterschiedlichen Gefechtsberichte über die Einnahme der Brücke in Dnepropetrovsk zu ganz unterschiedlichen Schlüssen über die Wichtigkeit von Personen und Einheiten kommen.

20 OKH, Kriegstagebücher (wie Anm. 16), S. 1.

21 Ebd.

22 Ein gutes Beispiel hierfür bietet der an sich sehr interessante Zusatz »Dnjepropetrowsk « zum Tätigkeitsbericht des Armeepionierführers der Panzergruppe 1 über die Stadt Dnepropetrovsk, der viele Aufklärungsfotos, Planskizzen und Notizen umfasst, die aber mangels Beschriftung schwer einzuordnen sind. Panzergruppe 1, Dnjepropetrowsk, Anl. 22 zum Tätigkeitsbericht 21.4.-15.12.1941, BA-MA, RH 21-1/214.

23 60. Infanteriedivision (mot.)/ Abt. Ia, KTB Nr. 3/Teil 2, BA-MA, RH 26-60/27.

24 13. Pz.Div./Abt. Ia, KTB Nr. 5, BA-MA, RH 27-13/12.

25 60. Infanteriedivision (mot.)/IVb, Tätigkeitsbericht für die Zeit vom 26.8. bis 17.11.1941, BA-MA, RH 26-60/71, Bl. 377. 
chronologisch den Verlauf der Operationen aus seiner Sicht. Dafür gehört er zu den wenigen, die klare Worte über die aus ihrer Sicht herrschenden Missstände finden, in diesem Falle insbesondere über die ungenügende Zusammenarbeit mit der Luftwaffe und der Waffen-SS ${ }^{26}$.

Die obengenannten Punkte tragen zur Charakterisierung des KTB als Quelle bei. Sie deuten nur auf einige wenige Aspekte hin, die hinsichtlich der KTBs für die historische Forschung von Bedeutung sind.

\section{Blitzkrieg und Städte}

Wie schon eingangs erwähnt, handelt es sich bei der Operation »Barbarossa« um den einzigen als Blitzkrieg geplanten Feldzug. Frieser definiert den Blitzkrieg taktisch-operativ als »den konzentrierten Einsatz von Panzerwaffe und Luftwaffe, um den Gegner durch Überraschung und Schnelligkeit zu verwirren und ihn nach erfolgtem Durchbruch mit weiträumigen Vorstößen zu umfassen. Ziel ist die rasche Niederwerfung des Gegners ${ }^{27}$. « Dieser Definition folgend sollen nun die Träger des Kampfes - Panzer und taktische Luftwaffe - sowie ihre Hauptstärke - Beweglichkeit - in Zusammenhang mit dem Stadtkampf gesetzt werden.

Speerspitze des Angriffs am Boden bildeten die Panzerdivisionen und die motorisierten Infanteriedivisionen. Ihre Vorstöße folgten zumeist den Straßen, da erstens auch Panzer und Halbkettenfahrzeuge auf Straßen schneller und mit geringerem Verbrauch an Treibstoff und Ersatzteilen vorankamen und zweitens nicht unerhebliche Teile dieser Verbände mit nur bedingt geländefähigen Fahrzeugen ausgerüstet waren. Dies betraf insbesondere die rückwärtigen Einheiten und die speziell für den Russlandfeldzug formierten Versorgungskolonnen. Das russische Straßennetz, und dies gilt besonders für die wenigen befestigten Straßen, war aber wegen der schieren Größe des Landes vor allem ein Netz von Straßen zwischen Städten, d.h. Knotenund Endpunkte dieser Straßen lagen in den Städten ${ }^{28}$. Dasselbe galt weitgehend auch für Brücken, und hier wiederum in besonderem Maße für panzertragende Brücken. Ein schneller Vormarsch der Panzerverbände konnte also in den spezifischen geografischen Verhältnissen Russlands die Städte nicht aussparen. Dies galt umso mehr für die Versorgungseinheiten ohne geländegängige Fahrzeuge.

Beim schnellen Vormarsch der ersten Wochen gegen eine überraschte Rote Armee gelang die Einnahme wichtiger Städte oft rasch und ohne großen Widerstand, so wie es die Idee des Blitzkrieges vorsah. Ein gutes Beispiel hierfür ist die Besetzung von Dünaburg ${ }^{29}$.

Doch mit zunehmender Dauer des Feldzuges verstärkte sich der russische Widerstand und im gleichen Maße nahmen auch die deutschen Kampfmittel ab. Dies

Panzergruppe 1/IVb, Tätigkeitsbericht vom 22.6.1941-1.4.1942, S. 23, BA-MA, RH 21-1/334. Frieser, Blitzkrieg-Legende (wie Anm. 1), S. 8. Frieser bringt auch eine strategische Interpretation des »Blitzkrieges (S. 9), weist jedoch darauf hin, dass dem Westfeldzug 1940 keine strategische "Blitzkrieg «-Planung zugrunde gelegen habe.

Dies wird besonders deutlich in den Kartenbeständen der untersuchten Einheiten, z.B. BA-MA, RH 24-3/63k für das III. Armeekorps (mot.).

Der Angriff auf die Sowjetunion (wie Anm. 2), S. 463 (Beitrag Klink). 
führte dann zu Situationen wie der Schlacht von Dnepropetrovsk. Die deutschen Panzerspitzen, in diesem Falle das III. Armeekorps (mot.) sollte in der Folge der Umfassungschlacht bei Uman Brückenköpfe über den Dnepr bilden. Dieser an seinem Unterlauf bis zu einem Kilometer breite Fluss bildete ein veritables Hindernis, dessen Wert durch die Sprengung von Dämmen noch erhöht worden war. Eine Umgehung der Stadt war wegen fehlendem Brückenmaterial und Nachschubmangel unmöglich ${ }^{30}$. Die einzigen drei Brücken, die in diesem Raum den Fluss querten, lagen in Dnepropetrovsk. Vorerst kämpften sich die Divisionen zwischen dem 15. und 23. August 1941 durch mehrere Verteidigungslinien an die Stadt heran. Bereits diese Kämpfe forderten erhebliche Verluste. Da die Infanterieverbände entweder noch mit der Säuberung des Kessels von Uman beschäftigt oder erst im Anmarsch waren, mussten die Panzerverbände selbst die Stadt erobern.

Am 24. August traten dann die 13. Panzerdivision und die 60. Infanteriedivision (mot.) zum Sturm auf die Stadt an, mit dem Ziel die Brücken unzerstört in die Hand zu bekommen und einen Brückenkopf zu bilden. Dies obwohl Panzerverbände für die Eroberung einer Stadt eigentlich ungeeignet sind. Die besonders auf Beweglichkeit getrimmten Verbände mussten in einem Gelände kämpfen, dass viele Möglichkeiten bot, die Beweglichkeit einzuschränken. Negativ bemerkbar machten sich auch die verhältnismäßig schwachen Infanterieverbände der schnellen Divisionen, die nur über vier Infanteriebataillone verfügten (gegenüber neun Infanteriebataillonen in der Infanteriedivision). Ein zusätzliches Bataillon Infanterie bestand im Kradschützenbataillon, aber auch dieses war eher für die schnelle Bewegung im offenen Gelände geschaffen worden. Einen Teil dieser Schwäche konnte durch den Einsatz kleiner Panzereinheiten (Züge oder Kompanien), die den Kampfgruppen als mobile Feuereinheiten zugewiesen waren, wettgemacht werden ${ }^{31}$. Im Gegensatz zu gezogenen Geschützen mit ungepanzerten Zugmaschinen hatten sie den Vorteil, Stützpunkte relativ geschützt zu bekämpfen, da die Panzerung wenigstens vor Gewehrfeuer schützte. Bei der Bekämpfung von Stützpunkten erwiesen sich insbesondere die für den Kampf Panzer gegen Panzer ungeeigneten Geschütze als brauchbar ${ }^{32}$. Zusätzlich waren die Panzer mit ein bis zwei Maschinengewehren ausgerüstet. Allerdings musste die Infanterie die Panzer gut schützen, da kaum ein Gelände so viele Bekämpfungsmöglichkeiten gegen Panzer bietet wie die Stadt. Dies wurde verstärkt durch die relative Schwäche der deutschen Panzerungen ${ }^{33}$. Mit den verschiedenen Panzerbüchsen verfügte die russische Infanterie sehr schnell über wirksame Panzerabwehrwaffen, die 1941 alle vorhandenen deutschen Panzer durchschlagen konnten ${ }^{34}$. Die Stadt bot aufgrund der Tarnmöglichkeiten zusätzlich

30 Vgl. hierzu: Ebd., S. 968-971 (Beitrag Müller), wo er auch feststellt, dass sich die ursprünglich Annahme, dass die Heeresgruppe Süd auf ihrem Vormarsch bis an den Dnepr heran ausreichend versorgt werden könne, als richtig erwies.

31 Vgl. hierzu: Gefechtsbericht des 2. Zuges 8. Kompanie (Lt. Roth) während der Unterstellung unter das I. Bataillon/Schützenregiment 93 vom 24.8.-25.8.1941, S. 2, BA-MA, RH 27-13/46 (Gefechtsbericht 2./8. Kp.).

32 Die 20-mm-Maschinenkanone des Panzer II wirkte als überschweres Maschinengewehr zum Niederhalten und die kurze 75-mm-Kanone des Panzer IV konnte Holz- und Steinbauten wirkungsvoll beschießen.

33 Frieser, Blitzkrieg-Legende (wie Anm. 1), S. $51 \mathrm{f}$.

34 Zur Entwicklung und Wirkung sowjetischer Panzerbüchsen: Reiner Lidschun und Günther Wollert, Infanteriewaffen gestern (1918-1945). Illustrierte Enzyklopädie der Infanteriewaffen aus aller Welt, 2 Bde, 3. Aufl., Berlin 1998, Bd 2, S. 459-464. 
die Möglichkeit, Panzernahbekämpfungsmittel wie Molotow-Cocktails, Minen und Handgranaten, mit guten Chancen zum Einsatz zu bringen.

Allein waren die Panzer also keine geeignete Waffe für den Stadtkampf. Als Unterstützung für die gemischten Kampfgruppen, die als Folge der Erfahrungen im Frankreichfeldzug zum Einsatz kamen ${ }^{35}$, konnten sie aber eine Verstärkung darstellen. Eminent wichtig dabei war die Zusammenarbeit der verschiedenen Waffengattungen, die bereits von der Reichswehr zu einer zentralen Doktrin erhoben worden war. Hierin lag einer der größten taktischen Vorteile der Wehrmacht gegenüber ihren Gegnern ${ }^{36}$.

Die zweite große Waffe des Blitzkrieges, die taktische Luftwaffe, konnte bei der Eroberung von Städten nur bedingt Unterstützung leisten. Eine direkte Unterstützung der angreifenden Bodentruppen kam aufgrund der engen Verzahnung ohne erhebliche Gefährdung der eigenen Truppe nicht in Frage. Sie konnte aber im Vorfeld der Kämpfe die Verteidigungsanlagen der Stadt attackieren. Dabei müssen allerdings drei Punkte berücksichtigt werden. Erstens waren Städte als Bevölkerungs- und Rüstungszentren oft auch Zentren der Luftverteidigung. So war beispielsweise der Luftraum um Moskau von rund 1000 Fliegerabwehrgeschützen und $585 \mathrm{Jagdmaschinen}$ geschüt $\mathrm{t}^{37}$. Zweitens boten die durch Luftangriffe zerstörten Gebäude oft neue Verteidigungsmöglichkeiten und schränkten die Durchfahrtsmöglichkeiten ein. Deshalb war die Bekämpfung von Artilleriestellungen und anmarschierenden Reserven erfolgversprechender. Drittens mangelte es der deutschen Luftwaffe zunehmend an Flugzeugen, um solche Aufträge durchzuführen ${ }^{38}$. Dies wird gerade bei Dnepropetrovsk ersichtlich, wo die russische Luftwaffe Tag und Nacht Angriffe auf die Brücke und die deutschen rückwärtigen Dienste fliegen konnte, weil die deutsche Luftwaffe neben ihrem Schwerpunkt in der Umfassungschlacht bei Kiev nicht mehr genügend Flugzeuge zur Unterstützung bei Dnepropetrovsk stellen konnte.

Ein weiteres Merkmal des Blitzkrieges war die Überraschung, und zwar sowohl auf der strategischen als auch auf der taktischen Ebene, im zeitlichen wie im örtlichen Sinne. Wie schon erwähnt konnte aufgrund der Überraschung die handstreichartige Einnahme einer Stadt gelingen. Solche Handstreiche konnten aus Sicht des Angreifers allerdings auch blutig enden, wenn das Überraschungsmoment verloren ging und es dem Gegner gelang, sich zu fassen. Dabei darf man nicht vergessen, dass gerade das Kampffeld Stadt dem Verteidiger gute Möglichkeiten zu einer raschen, improvisierten Verteidigung bietet ${ }^{39}$. Hierin liegt die wohl größte

35 Vgl, hierzu: Auswertung der taktischen Erfahrungen der Infanterie im Westen, $\mathrm{OKH}$ Nr. 1900/40 geh., BA-MA, RS-4/1234, Leibstandarte SS »Adolf Hitler « (LSSAH), Akten der LSSAH, Anl. II/121.

36 Vgl. Frieser, Blitzkrieg-Legende (wie Anm. 1), S. 426 f. und Robert M. Citino, The Path to Blitzkrieg. Doctrine and Training in the German Army, 1920-1939, Boulder, CO 1998, S. 15 f., für die Entwicklung des Denkens: Corum, The Roots of Blitzkrieg (wie Anm. 10), S. 7 f. und S. 74 f.

37 Der Angriff auf die Sowjetunion (wie Anm. 2), S. 692 (Beitrag Boog).

38 Ebd., S. 699 und 702, bezüglich Luftaufklärung: Ebd., S. 303.

39 In besonderer Weise bekamen dies die englischen Fallschirmjäger beim Versuch, zu ihren Kameraden am nördlichen Brückenende in Arnheim durchzudringen, zu spüren, als sie von rasch zusammengezogenen, bunt zusammengewürfelten deutschen Verbänden (Kampfgruppe Spindler) in den Vororten von Arnheim unter schweren Verlusten gestoppt 
Gefahr für einen Handstreich auf eine Stadt, der sich in der Folge zu einer ausgewachsenen und ressourcenverschlingenden Stadtschlacht entwickeln konnte ${ }^{40}$.

Insgesamt gesehen boten Städte für das "semimoderne «41 deutsche Heer in den spezifischen verkehrsgeografischen Verhältnissen Russlands großes Problempotenzial, was allerdings im Vorfeld, wie es scheint, zu wenig bedacht wurde.

\section{Vernichtungskrieg und Städte am Beispiel Barbarossa}

Die Zielsetzungen bezüglich der Städte im Vernichtungskrieg wurden vor allem in der jüngeren Forschung eingehend diskutiert ${ }^{42}$. Deshalb sollen hier nur die Grundlinien nachvollzogen werden, um mit den vorangegangenen Überlegungen zum Blitzkrieg den Gegensatz dieser beiden Konzepte hinsichtlich der Städte aufzuzeigen.

Der deutsche Vernichtungskrieg tangierte die Städte vor allem über zwei Linien: Hungerstrategie und vollständige Zerstörung von Großstädten. Allerdings gilt es vorwegzunehmen, dass Vieles hinsichtlich des Vernichtungskrieges vor Beginn der Operation nicht zu Ende gedacht war. Das zeigt sich insbesondere am Beispiel Leningrad, in der Diskussion um den Umgang mit etwaigen Hungerflüchtlingen ${ }^{43}$.

Im Rahmen der Hungerstrategie sollten die Überschussgebiete der Ukraine vom Rest der zu besetzenden sowjetischen Gebiete getrennt werden. Die Bevölkerung in diesen Restgebieten, namentlich der Waldzonen Mittel- und Nordrusslands sowie eben den Großstädten sollten durch eine Hungerpolitik entvölkert werden. Kalkuliert wurde dabei mit bis zu dreißig Millionen Hungertoten. Die Städte gerieten aber auch wegen ihrer nach deutschen Vorstellungen besonders kommunistischen Bevölkerung ins Visier ${ }^{44}$. Umgesetzt wurde diese Hungerpolitik dann allerdings ganz unterschiedlich ${ }^{45}$. Immer wieder kamen dabei Vorschläge zum Tragen, die darauf hinausliefen, die Städte abzuriegeln und auszuhungern,

wurden. Dieser rasch gebildete Sperrriegel, der stark durch das überbaute Gelände bevorteilt war, wurde als wichtiger Baustein des deutschen Sieges bei Arnheim identifiziert. Robert J. Kershaw, Arnheim ' 44 - Im September fällt kein Schnee. Die gescheiterte Luftlandung bei Arnheim, Stuttgart 2002, S. 103-108.

40 Städte bieten dem Verteidiger viele Vorteile, die die Erfolgschancen solcher Handstreiche herabsetzen. Es wäre deshalb interessant zu untersuchen, wie viele Stadtkämpfe ihren Ursprung in einem misslungenen Handstreich haben.

41 Der Begriff stammt von Theodor Ropp, vgl. hierzu Frieser, Blitzkrieg-Legende (wie Anm. 1), S. 37. Der Begriff beschreibt das Nebeneinander von vollmotorisierten Panzerdivisionen und zu Fuß marschierenden Infanteriedivisionen.

42 Jörg Ganzenmüller, Das belagerte Leningrad 1941-1944. Die Stadt in den Strategien von Angreifern und Verteidigern, Paderborn 2005; Christian Gerlach, Krieg, Ernährung, Völkermord. Forschungen zur deutschen Vernichtungspolitik im Zweiten Weltkrieg, Hamburg 1998; Der Angriff auf die Sowjetunion (wie Anm. 2), S. 1002-1015 (Beitrag Müller). Vgl. hierzu auch: Gert C. Lübbers, Die 6. Armee und die Zivilbevölkerung von Stalingrad. In: Vierteljahrshefte für Zeitgeschichte, 54 (2006), 1, S. 87-123. Dieser Artikel konnte leider nicht mehr ausgewertet werden.

43 Vgl. hierzu: Ganzenmüller, Das belagerte Leningrad (wie Anm. 42), S. 70-78.

44 Der Angriff auf die Sowjetunion (wie Anm. 2), S. 1013 (Beitrag Müller).

45 Ebd., S. 1002-1015. 
oft mit der vorgeschobenen Begründung, die eigenen Truppen schonen zu wollen. Die praktische Umsetzung entpuppte sich aber als äußerst kompliziert, auch unter dem Gesichtspunkt der Verkehrswege, die durch ein solches Vorgehen nicht nutzbar gewesen wären. Umgesetzt wurde diese Aushungerungsstrategie nur im Falle Leningrads. Auch hier war die praktische Umsetzung allerdings mit vielen Fragezeichen versehen ${ }^{46}$. Außerdem muss auf die Randlage Leningrads hingewiesen werden, die die Stadt für die weiteren deutschen Eroberungen als Knotenpunkt relativ unwichtig machte. Dieser Faktor darf bei der Diskussion um die Belagerung nicht übersehen werden. Ob nämlich ein ähnliches Vorgehen bei Moskau angesichts der logistischen Spannungen möglich gewesen wäre, ist äußerst fraglich. Zumindest die Vororte hätten besetzt werden müssen, um weiträumige Umfahrungen oder umfassende Ausbauten der Verkehrswege zu verhindern. Außerdem band jede Art des Aushungerns durch Belagerung verhältnismäßig viele Truppen. Die Hungerstrategie schuf also einen Gegensatz zu den Notwendigkeiten des Blitzkrieges in Bezug auf die Städte.

Die systematische Vernichtung ganzer Städte - geplant war sie insbesondere bei Moskau und Leningrad - stand in engem Zusammenhang mit der Hungerstrategie. Die bereits bei der Belagerung beginnende Zerstörung der Städte sollte nicht nur der Hungerstrategie Vorschub leisten, sondern auch die Einnahme erleichtern. Für wirksame Angriffe war aber entweder eine erhebliche Bomberflotte oder massierte schwere Artillerie mit der entsprechenden Reichweite erforderlich ${ }^{47}$. Die deutsche Bomberflotte, mit nur sehr geringen strategischen Kapazitäten, war schon zu Beginn des Russlandfeldzuges zu klein, und die rapide abnehmenden Zahlen verhinderten von Beginn an eine entsprechende Offensive zur Vernichtung von Städten, zumal mit Moskau und Leningrad zwei Ziele von erheblichem Umfang zu vernichten gewesen wären, ganz abgesehen von anderen Städten. Bei der schweren Artillerie lagen die Probleme etwas anders. Diese musste zuerst herangeschafft werden, was sich bei den äußerst beschränkten Transportkapazitäten negativ auf die Versorgungslage ausgewirkt hätte. Zusätzlich musste noch Munition im entsprechenden Umfang herangeführt werden. Dies wurde durch die große Typenvielfalt an Munition weiter erschwert. Die Konzentration an schweren Geschützen erforderte weiter eine entsprechende Sicherung, v.a. gegen Luftangriffe, was entweder weitere Transportkapazitäten benötigte oder die Frontverbände schwächte. Außerdem mussten genügend Stellungen vorhanden sein, was beim wenig tragfähigen Grund im Raum Leningrad erhebliche Probleme bereitete.

Es passt gut in das bis jetzt gezeichnete Bild des Vernichtungskriegs, dass die Zivilbevölkerung kaum ein Thema in den Kriegstagebüchern ist. Gemeinhin lassen sich in den Tagebüchern vor allem zwei Muster finden: Russische Zivilisten als Opfer des Kommunismus und als potenzielle Partisanen. Die erste Perspektive tritt in den untersuchten Tagebüchern oft rückblickend auf und hat wohl auch starken Rechtfertigungscharakter ${ }^{48}$. Deutlicher ist die Sicht auf die russische Zivilisten als potenzielle Partisanen oder insbesondere in Städten als Aufständische, gegen

46 Ganzenmüller, Das belagerte Leningrad (wie Anm. 42), S. 32-53.

47 Dies wird im Falle Leningrad besonders deutlich, da es nicht gelang, den Belagerungsring nahe genug an die Stadt zu legen, um sie in die Reichweite der Standardgeschütze zu bringen. Ebd., S. 30.

48 III. Armeekorps (mot.)/Abt. Ia, KTB Nr. 6: III. Pz.Korps - Vom Bug zum Don, S. 15, BAMA, RH 24-3/50. 
die mit aller Härte vorgegangen werden muss. Ein typisches Beispiel im Zusammenhang mit Dnepropetrovsk bildet der russische Artilleriebeschuss, der vermutlich von im Stadtgebiet zurückgelassenen Artilleriebeobachtern geleitet wurde ${ }^{49}$. Dieser Beschuss wirkte mit solcher Präzision auf die deutschen Stellungen und die einzige Brücke, dass das mit dem Brückenerhalt beauftragte Pionierbataillon 160 anregte, »dass das jenseitige Ufer mindestens in einer Breite von einem Kilometer und das diesseitige Ufer in einer Breite von $500 \mathrm{~m}$ von allen Zivilisten geräumt wird und jeder diese Sperrzone betretende Zivilist sofort niederzuschießen ist ${ }^{50}$." Diese drastische Maßnahme wurden aber vorerst nicht umgesetzt, wahrscheinlich mangels Truppen. Erst am 21. September 1941 findet sich in den Akten des Ic des III. Armeekorps (mot.) ein direkter Hinweis auf die Umsetzung von härteren Maßnahmen ${ }^{51}$. Dort wird ein strenges Durchgreifen gefordert, das auch das Nehmen von Geiseln und deren Erschießsung beinhaltet, falls der gezielte Artilleriebeschuss nicht aufhören sollte. Dies zeigt auf, wie wenig Rücksicht die Truppe auf die Zivilbevölkerung nahm ${ }^{52}$.

\section{Taktik und Führung deutscher Truppen im Stadtkampf}

Nun gilt es zu untersuchen, wie gut die deutsche Wehrmacht im taktischen Sinne auf das Kampfgelände Stadt vorbereitet war. Dies auch vor dem Hintergrund der oft kolportierten These, die Wehrmacht sei in Stalingrad an diesem für sie ungewohnten Kampfgelände gescheitert. Zuerst sollen an dieser Stelle die spezifischen Probleme des Stadtkampfes identifiziert werden:

49 "Da eine Feuerlenkung aus der Luft bei der heutigen Wetterlage nicht in Frage kommt, eine Leitung des Feuers durch die Art des Einschießens deutlich erkennbar erschien, kann nur eine Feuerlenkung durch Erdbeobachtung von einem Ufer erfolgt sein.« Pionierbataillon 160, Betr. Lenkung des feindl. Art.-Feuers durch Spione hinter der Front, in: Panzergruppe 1, Dnjepropetrowsk, Anl. 22 zum Tätigkeitsbericht 21.4.-15.12.1941, BA-MA, RH 21-1/214 (Pionierbataillon 160, Lenkung Artilleriefeuer).

50 Ebd.

51 Schreiben Ic III. A.K. (mot.) an Befehlshaber rückwärtiges Heeresgebiet Süd vom 21.9.1941, BA-MA, RH 24-3/135, Anl. 180. Zu diesem Zeitpunkt standen auch mehr Truppen zur Verfügung, weil die Kämpfe um den Brückenkopf zum Stillstand gekommen waren.

52 Ein weiteres Beispiel für das harte Vorgehen bietet der Befehl zur Entwaffnung der Bevölkerung. "Auf Befehl des Führers ist die Entwaffnung der Bevölkerung mit aller Schärfe zu beginnen und [...], wenn nötig mit den brutalsten Mitteln, restlos durchzuführen.« Zu diesen Mitteln gehörten wiederum die Geiselnahme und die Erschießung von Gefangenen, aber auch das Niederbrennen ganzer Dörfer, falls die $»$ Beteiligung großer Teile der Bevölkerung möglich [sic!] scheint«, aber nur »sofern die Quartiermöglichkeiten der Truppe dadurch nicht beeinträchtigt werden«. Speziell erwähnt wird die Entwaffnung der Bevölkerung in den großen Städten, da man hier im Zusammenhang mit der Hungerstrategie und aufgrund der vermuteten stärkeren Indoktrinierung der Bevölkerung ein erhebliches Aufstandspotenzial zu erkennen vermeinte. Betr.: Entwaffnung der Bevölkerung, Nr. 3545/41, BA-MA, RH 24-3/135. 
1. Fragmentierung des Gefechts: Gebäude werden zu kleinen Festungen, und eine Schlacht löst sich in viele einzelne Kämpfe kleiner Gruppen um solche Festungen auf, was durch das unübersichtliche Gelände verstärkt wird ${ }^{53}$.

2. Kurze Kampfdistanzen: Die Bebauung verkürzt die Sicht- und die Wirkungsdistanzen der Waffen. Die Kampfeinheiten befinden sich näher am Gegner, weshalb indirektes Feuer nur bedingt zum Einsatz kommen kann, ohne die eigene Truppe zu gefährden ${ }^{54}$.

3. Eingeschränkte Bewegungsmöglichkeiten für Fahrzeuge: Ashworth subsumiert diesen Punkt unter dem Vorteil der Verteidigung. Da er aber auch den Verteidiger betreffen kann (Heranbringen von Reserven oder Einsatz von eigenen Panzern), nehme ich ihn als eigenen Punkt. Die an sich schon beschränkten Bewegungsmöglichkeiten können sich im Verlaufe des Gefechts durch zerstörte Gebäude und Brände noch weiter vermindern ${ }^{55}$.

4. Erschwerte Führung und Kommunikation: Dieser Faktor steht in enger Wechselwirkung mit dem Punkt 1. Die erschwerte Führung fördert das Dezentralisieren der Kommandogewalt. Dadurch wiederum erschwert sich die Führung auf den oberen Ebenen. Zusätzlich Probleme tauchen durch geländebedingte Einschränkungen in den Kommunikationsmöglichkeiten auf.

5. Mangelnde Ortskenntnis: Ein wichtiger Faktor, der bei Ashworth nur am Rande erwähnt wird ${ }^{56}$, aber in so komplexen Anlagen wie Städten besonders zum Tragen kommt. Dabei spielt es auch eine Rolle, dass sich das Gelände durch die Kampfhandlungen verändern kann. Außerdem können unterirdische Netzwerke (U-Bahnen, Kanalisationen) eine wichtige Rolle spielen.

6. Anwesenheit von Zivilisten: Diese bilden eine doppelte Herausforderung: Einerseits können sie die Optionen für die militärische Führung einschränken, falls größere zivile Verluste vermieden werden sollen, andererseits stellen sie eine Bedrohung in Form von Aufständen dar ${ }^{57}$.

7. Absorption von Ressourcen: Ashworth spricht in seiner Arbeit nur von »manpower", aber es ist klar, dass es auch zu einem großen Verbrauch an Versorgungsgütern und Munition kommt. Beachtet werden sollte auch die höhere psychische Belastung der Truppe ${ }^{58}$.

Der sechste Punkt wurde bereits im Abschnitt über den Vernichtungskrieg behandelt, dem siebten Punkt wird anschließend an diesen Abschnitt ein eigener gewidmet.

Kurz beantwortet werden kann die Frage, ob die Wehrmacht hinsichtlich »Barbarossa « spezifische Vorbereitungen für dieses Kampffeld in Form von Ausbildung, Waffenentwicklung, Spezialformationen oder der Entwicklung einer Doktrin

Ashworth, War and the City (wie Anm. 3), S. 117 f. Seiner Ansicht nach reduzieren die schlechten Beobachtungsmöglichkeiten auch die Wirkung des Gefechts der verbundenen Waffen, eine Ansicht, die den gängigen taktischen Vorstellungen des Stadtkampfes diametral entgegensteht. Wir werden in der Fallstudie nochmals auf diese Vorstellung zu sprechen kommen.

54 Ebd., S. 118 f.

55 Ebd., S. 120.

56 Ebd., S. 121

57 Ebd., S. 119 f. Ashworth betont hier zu Recht, dass es nicht so wichtig ist, ob in einer Stadt tatsächlich ein Aufstandspotenzial vorhanden ist, sondern vielmehr, wie dies von der einrückenden oder verteidigenden Armee wahrgenommen wird. 
vornahm. Dies geschah erst im Zuge der Schlacht bei Stalingrad ${ }^{59}$. Insbesondere hinsichtlich der Doktrin kann das nicht verwundern. Denn die H.Dv. 300 hält im Abschnitt »Ortsgefecht « lediglich fest: »Auch Städte und Großstädte können zum Kampfgebiet werden ${ }^{60} . "$ Es ist dies das einzige Mal, dass in den sechs Ziffern der Begriff "Stadt « auftaucht, womit davon ausgegangen werden kann, dass der hier als »Stadtkampf « bezeichnete Gefechtstyp von der Wehrmacht unter der Doktrin des Ortskampfes subsumiert wurde ${ }^{61}$. Nun stellt sich natürlich die Frage, ob dies nicht sinnvoll war und wo genau denn die Unterschiede zwischen Orts- und Stadtkampf liegen? Ich möchte dies im Folgenden anhand der deutschen Ortskampfdoktrin skizzieren und damit auch gerade die Frage nach der Zweckmäßigkeit bzw. den Grenzen dieser Doktrin beantworten.

Es ist vor allem die Größe von Städten, an denen diese Gleichstellung scheitert. So wird beispielsweise in der Ziffer Nr. 553 der H.Dv. 300 festgehalten, dass Ortschaften vermehrt den Beschuss feindlicher schwerer Waffen auf sich ziehen. Natürlich ziehen auch Städte Angriffe von weitreichenden Waffen auf sich, aber sie sind flächenmäßig so groß, dass zu ihrer vollständigen Abdeckung mit Feuer erhebliche Kräfte zur Verfügung stehen müssen. Dazu existieren in Städten Möglichkeiten für unterirdische Bewegung, beispielsweise durch U-Bahn-Schächte oder Kanalisationen, die durch Beschuss kaum mehr zu bedrohen sind. Ähnliches gilt auch für die. in der Ziffer 555 genannte Umgehung zwecks Angriff aus der Flanke und dem Rücken. Auch hier taucht wieder das Problem der Größe von Städten auf. Stalingrad mit seinen Vororten zieht sich als 40 Kilometer langer Gürtel die Wolga entlang ${ }^{62}$. Eine Umgehung nimmt hier schon operatives Ausmaß an und ist auch logistisch nicht einfach zu bewältigen. In dieselbe Problematik fällt die in der Dienstvorschrift geforderte rasche Säuberung der Ortschaft. In größeren Städten wären hierzu erhebliche Kräfte erforderlich gewesen, die den durch die vorangehenden Kämpfe ausgebrannten Einheiten eben gerade fehlten. Die Wirkung dieses Problems haben wir bereits oben anhand der in Dnepropetrovsk zurückgelassenen russischen Artilleriebeobachter gesehen.

Dies gilt im Waffenbereich mit der Entwicklung von Sturmgeschützen mit schwersten Mörsern, etwa dem >Brummbär ‘ und dem >Sturmtigerc. Auch hier kamen viele Projekte nicht über das Planungsstadium hinaus. Für eine Doktrin lassen sich erste Ansätze in Form eines Erfahrungsberichtes sogar erst 1945 nachweisen. Vgl. hierzu: Befehlshaber des Ersatzheeres; Stab/IV(Ausb.) Ia(1) Nr. 778/45 g.; Betr. Kampfführung in Großstädten (Erfahrungen aus Budapest), BA-MA, RH 12-1/65, Bl. 39.

60 H.Dv. 300 (wie Anm. 9), S. 225.

61 Dies wird insbesondere durch die Bewertung der russischen Felddienstordnung PU-25 nahegelegt: "Während unsere F[ührung] und G[efecht] die für Ortsgefechte zu beachtenden Grundsätze in 6 Ziffern erschöpfend behandelt, werden in der russischen Felddienstordnung allein 73 Ziffern für das Kapitel sKampf in großen Städten und für ein besonderes Kapitel 'Kampf um bewohnte Punkte` weitere 22 Ziffern verwendet. Neue militärische Gesichtspunkte enthalten alle diese Ziffern nicht, auch keine grundsätzlichen Abweichungen von unseren Ansichten. Selbstverständlichkeiten oder Fragen, die sich aus den allgemeinen taktischen Grundsätzen ergeben, werden in schematischer, breiter Form wiederholt; es verlohnt sich nicht, im einzelnen [sic!] darauf einzugehen. " Reichswehrministerium, Heeres-Statistische Abteilung, Die russische Felddienstordnung vom 11.6.1925 (PU-25) unter besonderer Berücksichtigung der Gefechtsgrundsätze, Berlin, den 1.6.1925, S. 16 (In: BA-MA, RH 12-2/59, Fremde Heere von 1.4.1925-14.4.1928, Nr. 275-294).

62 Duncan Anderson, Lloyd Clark und Stephen Walsh, Die Ostfront 1941-1945. Barbarossa, Stalingrad, Kursk und Berlin, Wien 2002, S. 95. 
Andererseits werden wichtige Beobachtungen angestellt, die für den Stadtkampf von besonderer Bedeutung sind. So beschreibt die Ziffer 554 gleich drei wichtige Herausforderungen des Stadtkampfes in aller Kürze: Schnelle Abnutzung der Kräfte, kurze Kampfdistanzen und das selbstständige Handeln der Unterführer, also Dezentralisation der Führung, als entscheidender Faktor. Dies vor allem deshalb, weil der Kampf nach Einbruch in die Ortschaft nicht mehr systematisch geführt werden konnte, sondern in viele kleine Einzelgefechte zerfiel. Folgerichtig wird sowohl die Zusammenstellung von gemischten Kampfgruppen aus verschiedenen Waffengattungen, ebenso wie ein Nachrücken der Artillerie, um im Direktbeschuss den Feind zu vernichten, empfohlen.

Gänzlich unerwähnt dagegen bleibt die Anwesenheit von Zivilisten, welche die Kampfhandlungen behindern können, die eingeschränkten Bewegungsmöglichkeiten mechanisierter Kampfverbände und die Problematik der mangelnden Ortskenntnis. Da diese Faktoren insbesondere in Großstädten zum Tragen kommen, in Ortschaften dagegen eine Nebenrolle spielen, wird deutlich, dass die deutsche Doktrin des Ortskampfes nur sehr bedingt auf den Stadtkampf anwendbar war.

Wenn die deutsche Wehrmacht eine nur unvollkommene Doktrin und keine speziellen Formationen oder Waffen für den Stadtkampf hatte, wie fochten dann die regulären Heeresverbände den Stadtkampf durch?

Dies soll hier beispielhaft am Divisionsbefehl Nr. 48 der 13. Panzerdivision vom 23. August 1941 für den Sturm auf die Stadt gezeigt werden ${ }^{63}$. Der Befehl nennt im Sinne der Auftragstaktik das Divisionsziel des Angriffs: Die Eisenbahnbrücke von Dnepropetrovsk.

Unter Punkt 4 folgt dann die Zusammenstellung der Kräfte. Die Division formierte zwei gemischte Kampfgruppen, deren Kern die beiden Schützenregimenter der Division bildeten, verstärkt durch jeweils einen Zug Panzer, eine Kompanie Panzerjäger, eine Batterie Artillerie und Pioniereinheiten als Stoß- und Minenräumtrupps. Die so zusammengestellten Kampfgruppen waren eine Mischung verschiedener Waffengattungen, die rasch und flexibel auf jede Situation reagieren konnte. Ihre starke infanteristische Ausprägung war für den zu erwartenden Kampf im unübersichtlichen Gelände einer Stadt wichtig, ebenso die Betonung spezieller Pionierstoßtrupps, die mit Sprengladungen und Flammenwerfern etwaige Hindernisse und Stützpunkte im Nahkampf vernichten konnten. Die Artilleriegeschütze boten verstärkte Feuerkraft, wiederum im Direktbeschuss ${ }^{64}$. Die Panzer gaben den Kampfgruppen weitere, insbesondere mobile Feuerkraft. Sie mussten allerdings ihrerseits von der Infanterie gut geschützt werden, da sie auf die kurzen Distanzen des Kampfes in einer Stadt verwundbar waren. Aber die Mischung von Panzer und Infanterie in dieser Art von Gelände konnte sich sehr bewähren, auch wenn es sich um einen Rückgriff auf die eigentlich veraltete und verpönte Idee des Panzers als Unterstützungsmittel der Infanterie handelte. Der Panzereinsatz muss aber auch Nr. 48). Interessanterweise spricht dieser Befehl mit keinem Wort die Zivilbevölkerung an, auch nicht als potenzielle Aufständische.

64 Ebd., S. 2. Bei genauerer Betrachtung fällt auf, dass es sich um die beiden Abteilungen mit den leichten Feldhaubitzen (1.F.H.) handelte. Im weiteren Verlauf des Befehls wurden der schweren Abteilung Feuerunterstützungsaufträge zugewiesen, wobei die Kanonenbatterie Störfeuer auf die Brücken zu schießen hatte, um ein Zurückfluten der gegnerischen Verbände zu erschweren. 
im Zusammenhang mit den bereits stark geschwächten Schützenregimentern gesehen werden, die jede Art Verstärkung dringend brauchen konnten. Die Zusammenstellung folgt der H.Dv. 300, die in der Ziffer 556 Pioniere und Artillerie den angreifenden Verbänden zuweist.

Der Einsatz solcher gemischter Kampfgruppen war (und ist) aber keine einfache Angelegenheit. Verschiedenartige Gefechtsgrundsätze, Unkenntnis der Stärken und Schwächen anderer Waffengattungen oder schlicht mangelndes Vertrauen unter Kommandeuren und Truppen machte den Einsatz derartiger Kampfgruppen zu einer Herausforderung. Aber gerade in solchen Kampfgruppen lag die Stärke des deutschen Heeres, insbesondere in den Panzerdivisionen, die selbst als solche gemischten Kampfgruppen formiert waren. Dabei spielt die seit der Reichswehrzeit verstärkt betonte Doktrin des Gefechts der verbunden Waffen und deren konsequente Umsetzung in der Ausbildung ebenso eine Rolle wie das Ersatzsystem ${ }^{65}$, welches zu einem engeren Verhältnis innerhalb der Einheiten führte.

Unter Punkt 5, der die Kampfführung für die Infanterie und Artillerie regelt, wird dann Folgendes befohlen:

»Für den zu erwartenden Kampf in Schluchten, Gärten und Häusern stellen beide Kampfgruppen genügend Stoßtrupps bereit. Schwere Inf. Waffen und Art. dicht heran. Einzelgeschütze bei vorderster Inf., Minensuch- und -räumtrupps bereithalten. Zum Durchstoßen nach erfolgtem Einbruch genügend Kräfte - auch aufgesessen - bereitstellen, um in ausreichender Stärke bis zum Dnjepr durchstoßen zu können ${ }^{66}$."

Das dichte Aufrücken der Geschütze trug dem Kampf auf kurze Distanzen Rechnung. Um aber die maximale Feuerkraft an den Feind zu bringen, war es sinnvoll auch die leichten Artilleriegeschütze so einzusetzen. Bei der engen Verzahnung und dem unübersichtlichen Kampffeld konnte die Artillerie kein wirksames indirektes Feuer mehr schießen, ohne die eigenen Truppen zu gefährden. Ähnliches gilt auch für die Panzerabwehrgeschütze, die nicht wie sonst zur Abschirmung weiter hinten eingesetzt werden konnten, da sie dort mangels Sicht gar nicht in den Kampf hätten eingreifen können.

Auch der Kampf in kleinen Trupps wird berücksichtigt. Hauptträger des Kampfes bis zum Einbruch waren nämlich die Stoßtrupps - kleine Kampfeinheiten, die gut ausgerüstet unabhängig voneinander kämpften.

Unter Punkt 6 werden dann die Truppen genannt, die sich zur Verfügung der Division hielten. Aus den Angriffskräften ausgeschieden wurden alle Truppenteile, deren Stärke in ihrer Beweglichkeit lag, welche im Stadtkampf kaum zum Tragen gekommen wäre ${ }^{67}$. Damit ist auch klar, dass die eingeschränkten Bewegungsmöglichkeiten erkannt wurden.

65 Für eine Evaluation des deutschen Ersatzsystems: Creveld, Fighting Power (wie Anm. 10), S. 74-76. Siehe auch Stephen G. Fritz, Frontsoldaten. The German Soldier in World War II, Lexington, KY 1995, S. 157 f.

66 13. Pz.Div./Abt. Ia, Divisionsbefehl Nr. 48 (wie Anm. 63), S. 2. Dies zeigt auf, dass die deutschen Truppen fälschlicherweise von einer Frontlinie ausgingen, nach deren Durchbruch die Stadt gefallen war. Gerade Stalingrad sollte aber zeigen, das solch lineares Denken sich eben nicht auf den Stadtkampf anwenden lässt.

67 Es sind dies vor allem das Panzerregiment, die Aufklärungsabteilung und das Kradschützenbataillon. 
Dieser Divisionsbefehl zeigt gut auf, dass die Truppen im Feld eine adäquate Taktik entwickelten, um dem Kampfgelände Stadt Herr zu werden. Und dies weist uns auf die andere Seite der Medaille hin: Wohl verfügte die Wehrmacht nicht über spezielle Voraussetzungen für den Stadtkampf, aber die Ausbildung und Betonung bestimmter taktischer Grundsätze (Gefecht der verbundenen Waffen) ermöglichten es den Feldeinheiten, ein neues, anspruchsvolles Gelände zu bewältigen. Der Divisionsbefehl zeigt aber auch gut auf, wie wenig die Panzerdivision sich für den Stadtkampf eignete, da sie weitgehend auf ihre Hauptwaffe verzichten musste.

Zusätzlich zu den taktischen Grundsätzen sind die Führungsgrundsätze »Führen von vorne« und »Führen durch Auftrag (Auftragstaktik) von Bedeutung. Sie entfalten besonders im unübersichtlichen Kampffeld Stadt ihre Wirkung ${ }^{68}$.

Beim »Führen von vorne konnte sich der Kommandant vor Ort ein Bild machen, direkt mit seinen Untergebenen die Lage besprechen und Befehle ausgeben ${ }^{69}$. Angesichts der technischen Kommunikationsschwierigkeiten war dies ein großer Vorteil.

Ein gutes Beispiel für das »Führen von vorne « und dessen Wirksamkeit bietet der Gefechtsbericht des Schützenregiments 66, das den Brückenkopf über den Dnepr um den Mittag des 25. August 1941 bildete $^{70}$ : Noch während der Regimentskommandeur (Oberst Rodt) die Division über seine Absicht, einen Brückenkopf zu bilden, informierte, traf der Kommandierende General des III. Armeekorps (mot.) ein. Die beiden gingen zusammen zum Dnepr, der die Frontlinien bildete, wo sie auch die Führer des Artillerieregiments 13 und des Infanterieregiments 120 (mot.) der 60. Infanteriedivision (mot.) trafen. Um 10.50 Uhr hatte man die Entscheidung zum Übersetzen getroffen, welche dann sehr rasch in die Tat umgesetzt wurde. Um 12.30 Uhr hatte das I. Bataillon des Schützenregiments 66 die Floßsbrücke überschritten, um 13.15 Uhr war auch das II. Bataillon am Nordufer. Der an der Spitze des 43. Kradschützenbataillons marschierende Kommandeur wurde angewiesen, gleich hinter dem II. Bataillon zu folgen. Oberst Rodt und sein Gefechtsstand überschritten die Brücke hinter der verstärkten 1. Kompanie, also gerade hinter der Spitzenkompanie. Bereits um 14.15 Uhr traf im Brückenkopf der Stab der Schützenbrigade mit dem Brigadekommandeur, Oberst Herr, ein, der vorerst als Kommandant des Brückenkopfes die Führung aller übergesetzten Truppen übernahm, unabhängig ihrer Divisionszugehörigkeit ${ }^{71}$.

Die Anwesenheit dieser Führer hatte neben der Flexibilität auch noch einen weiteren, nicht zu unterschätzenden Vorteil, nämlich die moralische Stärkung der Truppe. Dies war gerade in der aktuellen Situation von großer Bedeutung, in der sich die Truppen des III. Armeekorps (mot.) seit zehn Tagen und mehr in Richtung Dnepropetrovsk vorkämpften und physisch wie psychisch erschöpft waren.

Das "Führen durch Auftrag « meint die als Auftragstaktik bekannt gewordene Doktrin, die dem Untergebenen das Ziel und die Mittel zuweist, ihm aber den Weg

68 Zu den beiden Führungsgrundsätzen: Frieser, Blitzkrieg-Legende (wie Anm. 1), S. 421-424. Zur Auftragstaktik auch: Creveld, Fighting Power (wie Anm. 10), S. 3-9 und S. 35-37.

69 Frieser, Blitzkrieg-Legende (wie Anm. 1), S. 423 f.

70 Schützenregiment 66, Gefechtsbericht über die Einnahme von Dnjepropetrowsk und die Bildung des Brückenkopfes am 25.8.1941, BA-MA, RH 27-13/46 (Gefechtsbericht S.R. 66).

71 Weitere Beispiele für die Anwesenheit höherer Kommandeure in der Frontlinie finden sich in: Gefechtsbericht 1./3./Pz.Pi. 4 (wie Anm. 14), Gefechtsbericht 2./8. Kp. (wie Anm. 31), S. 2. 
zum Ziel freistellt. Auf den Wert dieses Führungsgrundsatzes weist beispielsweise auch Manstein hin: »Die in keiner anderen Armee in gleichem Maße gepflegte Selbständigkeit der Führer bis zum Unterführer und bis zum Einzelkämpfer der Infanterie herab barg das Geheimnis des Erfolges ${ }^{72}$."

Diese Auftragstaktik musste aber in der Stadt von besonderer Bedeutung sein, da sie die Verantwortung und Führung in starkem Maße dezentralisierte, ebenso wie sich die Gefechte dezentralisierten. Dies betont auch die H.Dv. 300 für das Ortsgefecht: "[S]ein Ausgang hängt gewöhnlich von dem selbständigen Handeln der Unterführer $\mathrm{ab}^{73}$."Auch die Auftragstaktik lässt sich im Divisionsbefehl Nr. 48 nachweisen. In diesem Befehl wurden den Kampfgruppenführern das Ziel (die Inbesitznahme der westlichen Eisenbahnbrücke), die Mittel (die Einheiten jeder Kampfgruppe) und die Leitlinien des Vorgehens (in Form von Abschnittsgrenzen) vorgegeben. Außerdem war der Auftrag des vorgesetzten Verbandes (in diesem Falle der 13. Panzerdivision) angeführt, sodass die unterstellten Verbände bei einer günstigen Lageentwicklung im Sinne der Division handeln konnten. Die Division ließ aber den Kampfgruppen freie Hand bezüglich der Gliederung ihrer Truppen, in der Wahl des Vorgehens und der Schwerpunktbildung. Ein weiteres Beispiel war die Flussüberquerung des Schützenregiments $66^{74}$. Bemerkenswert ist hierbei, dass Oberst Rodt Rücksprache mit der Division nahm, damit diese ihm eine sich eventuell veränderte Auftragslage hätte mitteilen können. Dass aber Oberst Rodt nicht einfach auf das Einverständnis der Division wartete, sondern in dieser Zeit im Sinne des vorhandenen Auftrages handelte, zeigt sich an den zwei nachfolgenden Ereignissen: Einerseits am schnellen Übergang. Als um 10.45 Uhr die Bestätigung der Division eintraf, vergingen nur 90 Minuten, bis das I. Bataillon über die schmale Floßbrücke den Fluss überschritten hạtte. Und weitere 45 Minuten später war auch das II. Bataillon am anderen Ufer. Diese Leistung war nur möglich, weil die Truppe bereits vor dem Eintreffen der Bestätigung mit dem Bereitstellen begonnen hatte. Andererseits war auch Ausbesserungsmaterial bereitgestellt worden. Als nämlich die ersten Geschütze über die schmale Brücke gebracht werden sollten, kamen sie an einigen Stellen nicht weiter. Den nachrückenden Infanteristen konnte von diesem bereitgestellten Baumaterial mitgegeben werden, das sie im Vorbeiziehen an die entsprechenden Stellen brachten, wo es die Pioniere dann einbauten. So konnte die Brücke in kurzer Zeit behelfsmäßig ausgebessert werden, ohne dass der Übergang stockte.

Die Führungsgrundsätze trugen zur Überwindung der problematischen Lage im Bereich der Kommunikationsmittel bei. Die Kommunikation der Infanterieverbände auf taktischer Stufe lief über Drahtverbindungen oder Melder. In fast allen Berichten und Einträgen zur Schlacht von Dnepropetrovsk ist die Rede von unterbrochenen Leitungen, die beispielsweise das rechtzeitige Feuer der eigenen Artillerie verunmöglichten, oder Meldeläufern, die ihr Ziel nie erreichten ${ }^{75}$. Allerdings boten auch die Funkgeräte keine Gewähr für bessere Verbindungen, da ihre Reichweite

72 Erich von Manstein, Verlorene Siege, 12. Aufl., Bonn 1991, S. 57.

73 H.Dv. 300 (wie Anm. 9), S. 226.

74 Vgl. zum Folgenden: Gefechtsbericht S.R. 66 (wie Anm. 70).

75 Gefechtsbericht für die Zeit vom 11.8.-1.9.1941 (Artillerieregiment 13), BA-MA, RH 2713/46, Anlage III/114 (Gefechtsbericht A.R. 13); Gefechtsbericht für die Zeit vom 24.-26.8.1941 (II. Abteilung/A.R. 13), BA-MA, RH 27-13/46, Anlage III/143. 
im Stadtgelände durch die massiven Bauten oft stark reduziert war. So lag die Stärke der beiden Führungsgrundsätze darin, dass sie die problematische Kommunikationslage weitgehend ausgleichen konnten.

\section{Logistische Zwänge}

Jede Störung der von Anfang an labilen Logistik der Operation »Barbarossa« musste zwingend in die Katastrophe führen ${ }^{76}$. Deshalb müssen neben den operativen Implikationen des Stadtkampfes unbedingt auch die logistischen betrachtet werden. Und hier gilt es das Augenmerk auf zwei Punkte zu richten: Die speziellen geografischen Verhältnisse Russlands ${ }^{77}$ und den enormen Ressourcenverschleiß des Stadtkampfes.

Während in Westeuropa bereits im Zweiten Weltkrieg relativ dichte Verkehrsnetze existierten, welche die Umgehung einer Stadt auch auf Straßen ermöglichten, bestand das russischen Verkehrsnetz, wie wir bereits gesehen haben, weitgehend aus Verbindung zwischen Städten. Mochten auch Panzer und Halbkettenfahrzeuge querfeldein operieren, die Logistikverbände der Wehrmacht konnten es richt. Und die geländegängigsten Fahrzeuge blieben buchstäblich auf der Strecke, wenn sie keinen Treibstoff mehr hatten. So galt es die Straßen zu erobern, und mit ihnen die Knoten- und Endpunkte, also die Städte ${ }^{78}$. Doch gerade die Städte boten guten Schutz vor den deutschen Panzerspitzen. Noch wichtiger für die Versorgung der Wehrmacht war die Eisenbahn, die aber in Russland ebenfalls ein Netz von Strecken zwischen Städten bildete ${ }^{79}$.

Die zweite Verknüpfung von Stadtkampf und Logistik liegt in den erheblichen Menschenverlusten bzw. im immensen Materialverbrauch, der mit der Eroberung einer Stadt einherging. Dies wird deutlich bei der Betrachtung des Munitionsverbrauchs, der Ausfälle bei der Truppe und der zusätzlichen Belastung der Transporteinheiten in der Schlacht um Dnepropetrovsk.

Von besonderer Bedeutung war der Munitionsverbrauch. Gemäß dem Oberquartiermeister (Ib) der Panzergruppe 1 sollen im Kampf um Dnepropetrovsk bis zum 6. September 194140000 Tonnen Artilleriemunition verschossen worden $\operatorname{sein}^{80}$.

76 Zur Logistik der Operation »Barbarossa«: Martin van Creveld, Supplying War. Logistics from Wallenstein to Patton, Cambridge 1977, S. 142-180; Der Angriff auf die Sowjetunion (wie Anm. 2), S. 113-118 (Beitrag Müller); ebd., S. 168-189 (Beitrag Müller); ebd., S. 248-259 (Beitrag Klink); ebd., S. 959-988 (Beitrag Müller).

77 Vgl. hierzu auch den Abschnitt "Blitzkrieg .und Städte«.

78 Damit verbunden ist auch das Problem panzertragender Brücken, die oft ebenfalls in Städten zu finden waren.

79 Zu den grundsätzlichen Problemen der Eisenbahn als Hauptträger der Logistik im Blitzkrieg: Creveld, Supplying War (wie Anm. 76), S. 153 f. und besonders für die Heeresgruppe Süd: S. 163-166. Dort auch der wichtige Hinweis, dass Straßen und Eisenbahnen oft völlig unterschiedliche Routen nahmen, was dazu führte, dass die zum Umnageln und Reparieren angesetzten Trupps die Linien erst freikämpfen mussten.

80 Panzergruppe 1/O.Qu., KTB, 6.9.1941, BA-MA, RH 21-1/327. 
Trotz aller Vorbehalte gegenüber dieser Zahl ${ }^{81}$ deutet sie an, dass dieser Stadtkampf mehr Ressourcen verschlang, als der Wehrmacht zur Verfügung standen. Der hohe Verbrauch an Munition war Folge einer Artilleriekonzentration, wie sie in der Operation »Barbarossa " nur selten erreicht wurde. Eine Artilleriekarte der 60. Infanteriedivision (mot.) ${ }^{82}$ weist 17 Abteilungen auf. Nach Angaben des Ib der Panzergruppe 1 seien am 5. September 1941 sogar 25 Artillerieabteilungen im Kampf gewesen $^{83}$. Diese beiden Aufstellungen deuten bereits ein weiteres Problem für die Versorgung an: Die große Typen- und Kalibervielfalt der Wehrmacht ${ }^{84}$. Neben der üblichen Divisionsartillerie mit drei unterschiedlichen Munitionstypen waren mindestens drei italienische Abteilungen der Division "Pasubio" sowie zwei schwere Artillerieabteilungen des Korps anwesend ${ }^{85}$, die allesamt unterschiedliche Munition verschossen. Trotz erheblicher Zuführung schmolzen die Artilleriemunitionsbestände dahin, sodass beispielsweise die 198. Infanteriedivision ab dem 12. September nur noch 50 Prozent einer Erstausstattung zur Verfügung hatte ${ }^{86}$. Der Mangel an Munition aber hatte zur Folge, dass die sowjetische Artillerie nur ungenügend bekämpft werden konnte. Dies wiederum bewirkte hohe Verluste bei den deutschen Truppen $^{87}$.

Aber nicht nur die Artilleriemunition belastete die Versorgung, sondern der im Stadtkampf ebenfalls hohe Verbauch an Infanteriemunition, wozu auch die Munition für Granatwerfer und Infanteriegeschütze sowie Handgranaten zählten. Deutlich wird dies am Beispiel der 198. Infanteriedivision. Am 28. August 1941 meldete sie der Panzergruppe 1 in der abendlichen Versorgungslage, dass eine volle Erstausstattung an Munition vorhanden $\mathrm{se}^{88}$. Bereits am 6. September 1941 verfügte die Division trotz Zuführung über weniger als die Hälfte ihrer Erstausstattung an Infanteriemunition. Besonders gravierend war der Mangel bei den schweren Infanteriewaffen (Granatwerfer, Infanteriegeschütze). Die Divisionsführung reagierte darauf, indem sie die Kompetenz zum. Verschuss solcher Munition an sich zog ${ }^{89}$.

Die Zahl erscheint mir sehr hoch, wenn man bedenkt, dass die 13. Panzerdivision zwischen dem 19. August und dem 7. September insgesamt 661,9 Tonnen Artilleriemunition zugewiesen bekam. Vgl. hierzu: 13. Pz.Div./W.u.G, Tätigkeitsbericht 18.8.-7.9.1941, BAMA, RH 27-13/128 (Tätigkeitsbericht W.u.G. 13. Pz.Div.). Die Frage ist allerdings, ab welchem Zeitpunkt diese Rechnung beginnt und ob wirklich nur die Artilleriemunition darin enthalten ist. Unbestritten ist aber, dass der Munitionsverbrauch extrem hoch war, nach Angaben des W.u.G.-Offiziers allein für die 60: Infanteriedivision (mot.) fünf- bis sechsmal so hoch wie erwartet. Vgl. hierzu: 60. I.D. (mot.)/W.u.G., Betr.: Beitrag zum Kriegstagebuch, BA-MA, RH 26-60/71, Anlage 369 - Tätigkeitsbericht W.u.G. 60. I.D. (mot.).

82 60. I.D. (mot.)/ Abt. Ia, Artilleriegliederung 2.9.1941, Karten zum KTB Nr. 3 Abt. Ia, BAMA, RH 26-60/50k

83 Panzergruppe 1/O.Qu., KTB, 5.9.41, BA-MA, RH 21-1/327.

84 Einen guten Überblick über die problematische Ausrüstungslage der Wehrmacht bereits bei Angriffsbeginn gibt: Der Angriff auf die Sowjetunion (wie Anm. 2), S. 186 f. (Beitrag Müller).

85 731. (mot.) mit 15-cm-Schiffskanonen SKC 28 und 604. (mot.) mit 21-cm-Mörsern 18.

86 Eine volle Ausstattung betrug 250 Schuss pro 1.F.H und 275 Schuss pro 10-cm-Kan 18. 13. Pz.Div./W.u.G, Tätigkeitsbericht, 3.8.-15.8.1941, BA-MA, RH 27-13/127.

87 Alleine die 60. I.D. (mot.) gab ihre Verluste seit Beginn der Kämpfe im Brückenkopf mit täglich 100 Mann an. Fernschreiben 31.8.1941 der 60. I.D. (mot.) an III. A.K., BA-MA, RH 26-60/37.

88 198. I.D./Abt. Ib, Versorgungslage 28.8.1941, BA-MA, RH 26-198/93, Nr. 28.

89 Ebd., Munitionswesen, BA-MA, RH 26-198/91, Nr. 48. 
Damit wurde faktisch die Leitung des Feuers einzelner Geschütze Aufgabe des Divisionsstabes. Bestimmte Waffentypen konnten mangels Munition gar nicht mehr eingesetzt werden, so der im Stadtkampf besonders effektive 81-mm-Granatwerfer ${ }^{90}$. Auch bei den anderen Divisionen herrschte bald Munitionsknappheit für die schweren Infanteriewaffen ${ }^{91}$.

Diese Versorgungslücken traten auf, obwohl schon seit dem 21. August 1941 der überwiegende Teil der verfügbaren Nachschubmengen der Panzergruppe 1 in den Raum Dnepropetrovsk flossen ${ }^{92}$. Die Folge: Der Ib der Panzergruppe 1 sah sich veranlasst, beim Quartiermeister des III. Armeekorps (mot.) anzuordnen »den taktischen Einsatz der Artl. mit den versorgungsmäßigen Möglichkeiten in Einklang zu bringen ${ }^{93}$. So musste sich zeitweise auch in der Wehrmacht das sonst übliche operative Primat der logistischen Realität unterordnen.

Die neuere Forschung hat aufgezeigt, dass die logistischen Probleme in noch viel stärkerem Maße von den beschränkten Transportmöglichkeiten abhingen als vom grundsätzlichen Mangel an Versorgungsgütern ${ }^{94}$. Deutlich zeigt sich dies auch im Raum Dnepropetrovsk. So vermerkte am 20. August 1941 der Ib der Panzergruppe 1 , dass die

"Pz.Gr., die mit ihrem durch die starke Beanspruchung auf 60 Prozent reduzierten Transportraum neben 9 mot.- und Pz.Divn. [Panzerdivisionen] das XXXXIV. A.K., Ital. Exped. Korps [Italienisches Expeditionskorps] und das Ung. Schnellkorps [Ungarisches Schnellkorps] versorgen muss. Die Entfernung des Ausladebahnhofs Bialacerkiew [...] beträgt $350 \mathrm{~km}$. Der Quartiermeister kann unter diesen Umständen für eine reibungslose Versorgung nicht mehr garantieren ${ }^{95}$."

Noch deutlicher wird der Waffen- und Geräte (W.u.G.)-Offizier der 60. Infanteriedivision (mot.) in seinem Erfahrungsbericht. Er berichtet von Transportstrecken von durchschnittlich 200 Kilometer, in Extremfällen sogar 500 Kilometer - bei den in der Sowjetunion üblichen miserablen Straßenverhältnissen - die zwischen Ausladebahnhöfen und Front bewältigt werden mussten. Dies alleine bedeutete eine starke Belastung, aber kombiniert mit dem hohen Munitionsverbrauch und der sich rasant verschlechternden Fahrzeuglage musste dies zu einer erheblichen Krise führen. In seinem Bericht geht der W.u.G.-Offizier auch davon aus, dass bei voller Munitionslieferung die Versorgungsdienste innerhalb kürzester Zeit zusammengebrochen wären ${ }^{96}$.

Dies wird auch ersichtlich aus der täglichen Versorgungslage zwischen dem 23. und 25. September. An diesen Tagen werden die Bestände mit 0-1,8 \% der Erstausstattung angegeben. Auch in den folgenden Tagen steigt dieser Bestand nicht über $20 \%$ der Erstausstattung. 198. I.D./Abt. Ib, Versorgungslage 23., 24., 25.9.1941, BA-MA, RH 26-198/93. Für die 60. Infanteriedivision (mot.) zeigt dies der Bericht des W.u.G.-Offiziers sehr gut, Tätigkeitsbericht W.u.G. 60. I.D. (mot.) (wie Anm. 81). Für die 13. Panzerdivision finden sich einige Hinweise im KTB Nr. 5 Abt. Ib, BA-MA, RH 27-13/126 und im Tätigkeitsbericht W.u.G. 13. Pz.Div (wie Anm. 81).

92 Panzergruppe 1/O.Qu., KTB, 21.8.1941, BA-MA, RH 21-1/327.

93 Panzergruppe 1/O.Qu., KTB, 6.9.1941, BA-MA, RH 21-1/327.

94 Der Angriff auf die Sowjetunion (wie Anm. 2), S. 960-965 (Beitrag Müller).

95 Panzergruppe 1/O.Qu., KTB, 20.8.1941, BA-MA, RH 21-1/327.

96 Tätigkeitsbericht W.u.G. 60. I.D. (mot.) (wie Anm. 81). Dieser Bericht ist insbesondere deshalb wertvoll, weil er auf viele Detailprobleme der Versorgung hinweist. 
Standen also an sich schon nicht genügend Fahrzeuge für die Versorgung bereit, so wurden die Fahrzeugbestände durch Zusatzaufgaben und Ineffizienz weiter belastet.

Zu den Zusatzaufgaben gehörte vor allem das rasche Heranführen weiterer Kampftruppen. Im Falle Dnepropetrovsk finden sich zwei Beispiele, die den Umfang solcher Aktionen aufzeigen. Mit dem Einsetzen der sowjetischen Gegenangriffe und dem dringenden Bedarf nach frischen Infanteriekräften wurde die 198. Infanteriedivision ab dem 28. August 1941 motorisiert nach Dnepropetrovsk transportiert. Da die Division dies nicht mit eigenen Mitteln bewerkstelligen konnte, wurden von der 16. Panzerdivision und der Leibstandarte-SS »Adolf Hitler« (LSSAH), die sich beide in der Auffrischung befanden, je 200 Transportfahrzeuge herangezogen. Für die LSSAH bedeutete dies in der Folge, dass sie bis zum 10. September 1941 in ihrem Auffrischungsraum gebunden war, weil nahezu gleich viele Fahrzeuge, wie zur 198. Infanteriedivision übergingen, nicht einsatzfähig waren ${ }^{97}$. Außerdem führte dieser Transporteinsatz zu weiteren Ausfällen. Damit aber war die Auffrischungsphase hinsichtlich der Transportfahrzeuge für die LSSAH nutzlos ${ }^{98}$. Beim Antransport der 198. Infanteriedivision verzichtete man mangels Transportraum auf die Mitführung aller rückwärtigen Dienste, sodass sie den schon überlasteten Versorgungstruppen im Raum Dnepropetrovsk zur Last fiel. Das zweite Beispiel war der Antransport eines Pionierbataillons durch 144 Fahrzeuge der 13. Panzerdivision am 31. August 1941. Die schon angespannte Fahrzeuglage verschärfte sich durch solche Einsätze zusätzlich.

Dass aber auch Ineffizienz eine Rolle spielte, zeigt die Zuführung des Ersatzbataillons 1073, mit dem die 13. Panzerdivision einen Teil ihrer Verluste ersetzen sollte $^{99}$. Da am 27. August 1941 keine Fahrzeuge zur Verfügung standen, wurde nur ein Vorauskommando entsandt, welches das Bataillon in Belaja Cer'kov (Bialacerkiew) in Empfang nehmen sollte. "Nachdem inzwischen wieder einmal ein anderer Abholungsort für das Ersatz.Btl. befohlen worden ist «, wie der Tagebuchführer am 30. August 1941 nicht ohne Anflug von Frustration vermerkte, wurde auch das Abholkommando nach Pervomajsk entsandt. Als am nächsten Tag um 22.00 Uhr mit Belaja Cer'kov, das etwa 100 Kilometer von Pervomajsk entfernt liegt, erneut der Abholpunkt gewechselt wurde, folgte einer der raren Einträge mit deutlichen Worten:

»Hptm. [Hauptmann] Lieff fuhr auf Grund dieser dauernden Änderungen 2x zwischen Bialacerkow [entspricht Bialacerkiew] und Perwomaisk hin u. her. Wäre ihm - falls die Gestellung möglich gewesen wäre - die Abholkolonne sofort mitgegeben worden, so hätte diese ebenfalls $2 x$ die Strecke umsonst zurückgelegt. Betr.St.-Verbrauch [Betriebsstoffverbrauch] und Kfz.-Verschleiß wäre untragbar gewesen. Die Erfahrung, die die Truppe aus diesen Änderungen sammelt, können auf die Dauer zu lässiger Ausführung von Befehlen führen.« Diese Versorgungsprobleme wirkten sich auch auf die Sanitätsdienste aus. Diese standen aufgrund erheblicher Truppenverluste schon unter starkem Druck. Anhand MA, RS 3-1/24.

98 Vgl. hierzu auch: LSSAH, Bericht über den Einsatz der L.SS.A.H. vom 22.8.-22.9.1941, BAMA, RS 3-1/24.

99 Der folgende Teil ist dem KTB Nr. 5 Abt. Ib der 13. Panzerdivision, BA-MA, RH 27$13 / 126$, entnommen. Hervorhebung im Original. 
der Verlustliste der 198. Infanteriedivision kann man gut erkennen, welch ungeheure Ausfälle die Division in der Phase der intensiven Kämpfe am Stadtrand und um das Bahnhofs- und Kasernengelände am Ostrand von Dnepropetrovsk zu verzeichnen hatte. In den elf Tagen zwischen dem 31. August und dem 10. September 1941 hatte die Division 429 Tote zu beklagen, darunter 14 Offiziere. Dazu kamen 1172 Verwundete, inklusive 39 Offiziere ${ }^{100}$.

Auffallend ist der hohe Anteil an Toten, der zeitweise nahezu 30 Prozent der Ausfälle ausmachte. Neben den kurzen Kampfdistanzen des Stadtkampfes dürfte dies einen engen Zusammenhang mit den völlig überforderten Sanitätsdiensten haben. Da diese - wie die anderen rückwärtigen Dienste - mit relativ wenigen Fahrzeugen, geschweige denn geländegängigen, ausgestattet waren, konnten sie den vorrückenden Truppen nur mit Mühe folgen. Die Zuführung der Kampftruppen der 198. Infanteriedivision ohne ihre Sanitätsdienste brachte weitere Probleme. Dazu kam, dass die im Raum Dnepropetrovsk eingesetzten Sanitätseinheiten seit dem 13. August 1941 ohne Pause im Einsatz standen und bereits einen erheblichen Anfall von Verwundeten zu bewältigen hatten, sodass bereits von einer Überforderung gesprochen werden kann $^{101}$. Die Situation verschärfte sich seit dem Beginn der Kämpfe in der Stadt (24. August 1941) erheblich. Bald waren die Chirurgenteams so erschöpft, dass trotz weiterer Verwundeter ganze Tage hindurch pausiert werden musste, auch wegen der Gerätewartung.

Ein weiteres Problem war die Nähe zur Front. Hauptverbandsplätze sollten normalerweise sechs bis zehn Kilometer von der Front entfernt sein, Feldlazarette 15 bis 20 Kilometer. Tatsächlich befanden sich aber zwei Hauptverbandsplätze im Brückenkopf, der keine drei Kilometer Durchmesser hatte. Mindestens ein Feldlazarett war in der Stadt eingerichtet worden, was bestenfalls knapp zehn Kilometer Distanz zur Front ergab. In der Folge gerieten die Sanitätsstellen unter Artilleriebeschuss, dies führte zu Arbeitsunterbrechungen und Personalausfällen.

Nur durch ständigen Abtransport aus den Feldlazaretten konnte man der Lage überhaupt Herr werden, da in den ersten zehn Tagen im Durchschnitt mehr als 200 Verwundete anfielen. Wenn man bedenkt, dass ein Feldlazarett bei voller Ausstattung 200 Betten hatte, kann man leicht einschätzen, wie viele Tage es dauerte, bis die fünf eingesetzten Feldlazarette mit Verletzten gefüllt waren. Der ständige Abtransport verursachte allerdings weitere Probleme: Da die Krankenwagenkolonnen mit dem Antransport zu den Feldlazaretten völlig ausgelastet waren, mussten Versorgungsfahrzeuge auf den Leerfahrten zu den Depots. Verwundete mitnehmen. Diese Fahrzeuge waren aber in keiner Weise für den Verwundetentransport ausgestattet. Deshalb mussten ihnen Tragen und Decken mitgegeben werden, die aber selten zurückkamen. So herrschte nach wenigen Tagen Mangel an Decken und Tragen.

Die Zahlen basieren auf einer Zusammenstellung anhand der täglichen Versorgungslageberichte, BA-MA, RH 26-198/93, 198. Infanteriedivision, Mappe 4 zum KTB Nr. 5 der Abt. Ib, Nr. 28-57.

101 Dies zeigen insbesondere die Tätigkeitsberichte des IVb der 60. Infanteriedivision (mot.). Die folgenden Absätze beziehen sich, soweit nicht anders vermerkt, auf drei Berichte: 60. I.D. (mot.)/IVb, Tätigkeitsbericht für die Zeit vom 11.8.-25.8.1941, Anlage 377; 60. I.D. (mot.)/IVb, Tätigkeitsbericht für die Zeit vom 26.8.-17.11.1941, Anlage 375; 60. I.D. (mot.)/IVb, Bergung, Versorgung und Abtransport im Brückenkopf Dnjepropetrowsk, Anlage 376; alle drei in: BA-MA, RH 26-60/71. 
Allerdings müssen die verhältnismäßig hohen Anteile an Toten nicht nur mit der Überforderung der Sanitätsdienste zusammenhängen, sondern können in doppelter Hinsicht auch eine Überforderung der Truppe aufzeigen. Die völlig erschöpften Soldaten waren weniger aufmerksam, was zu mehr Verletzten und Toten führte. Und die erschöpften Verletzten verfügten über weniger Widerstandskräfte, um ihre Verwundungen zu überstehen ${ }^{102}$.

Die beiden logistischen Problemstellungen weisen auf einen Widerspruch hin. Einerseits war die Wehrmacht unter den verkehrsgeografischen Verhältnissen der Sowjetunion gezwungen, Städte einzunehmen, um in den Besitz der wichtigen Knotenpunkte und Flussübergänge zu gelangen, die die weitere logistische Voraussetzung für den Vormarsch bildeten. Andererseits aber war gerade die Einnahme dieser Städte, sofern ernsthaft verteidigt, mit einem erheblichen Aufwand an Material verbunden, sowie oft auch mit großen Verlusten an Menschenleben, Ressourcen also, die gerade durch den Blitzkrieg geschont werden sollten.

\section{Schlussbetrachtung}

In der Operation »Barbarossa « entstand eine für die Wehrmacht verhängnisvolle Verkettung: Unter den besonderen russischen Verhältnissen bildeten Städte als Knoten- und Endpunkte der Verkehrswege eben auch Knotenpunkte der Logistik, unabhängig davon ob auf der Straße oder Schiene. Diese galt es schnellstmöglich zu nehmen. Die der Masse der zu Fuß marschierenden Armee vorauseilenden motorisierten Infanterie- und Panzerdivisionen waren für die Einnahme von Städten aber mangels Infanterie ungeeignet, es sei denn, die Einnahme gelang durch Überraschung. War der Gegner aber zur Verteidigung vorbereitet oder konnte er sich im Verlauf eines Handstreichs wieder stabilisieren, dann mussten zeitaufwendig Infanterie und Artillerie herangebracht werden. In dringenden Fällen geschah dies mit Lastkraftwagen, die von überallher zusammengezogen wurden, was wiederum Auswirkungen auf die angespannte Logistik und Beweglichkeit der abgebenden Einheiten hatte. Kam die Infanterie zu Fuß heran und säuberte dann mühsam eine Stadt, so musste sich der Abstand zu den vorrückenden, deutlich schnelleren Panzerdivisionen weiter vergrößern, die außerdem zunehmend unter logistischen Problemen zu leiden hatten. Die Alternative bestand im Anhalten der schnellen Verbände, was dem Gegner das Sammeln erlaubte und der Grundidee des Blitzkrieges

102 Dies bestätigt sich bei einer Zusammenstellung der Sterbezahlen über den Zeitraum von der Verlegung bis zum Ausbruch aus dem Brückenkopf Ende September. Ab dem 10. September wurden die Kampfhandlungen seltener, gleichzeitig verfügte die Truppe nun über eine ausgebaute Stellung, musste also nicht dauernd noch schanzen, und konnte zusätzlich mit der Bildung einer Reserve beginnen, die im Rotationsprinzip den Soldaten 36 Stunden Ruhezeit verschaffen sollte. Von diesem Moment an sank auch der Anteil an Toten an den Gesamtverlusten. Vgl. zur Reservenbildung wie auch zum allgemeinen Erschöpfungszustand der Truppe am Beispiel der 198. Infanteriedivision: 198. I.D./Abt. Ia, KTB Nr. 6, BA-MA, RH 26-198/6 sowie Anlage 4 zu KTB Nr. 6 Abt. Ia, BA-MA, RH 26$198 / 10$, jeweils ab dem 1 . bis 30 . September. 
widersprach. In diesem Sinne führten Stadtkämpfe zur Vergrößerung der Kluft zwischen Infanterie- und Panzerverbänden.

Ein weiterer Widerspruch zu diesen militärischen Bedürfnissen der schnellen Einnahme bildeten die Ziele des Vernichtungskrieges, die eine Besetzung der Städte gerade nicht vorsahen, beziehungsweise erst nach der Vernichtung der Bevölkerung. Vorgeschoben wurde dabei das Argument der Ressourcenschonung, sei es bezüglich Nahrungsmittel, militärischer Versorgungsgüter oder auch Truppen, die sich, wie wir gesehen haben, in Stadtkämpfen besonders schnell abnutzten. Dass diese Argumente allerdings nicht gänzlich vorgeschoben waren, zeigt der Fall Dnepropetrovsk.

Andererseits - und dies überrascht angesichts der im Zusammenhang mit Stalingrad oft kolportierten These, dass die deutschen Truppen auf den Stadtkampf völlig unvorbereitet gewesen seien - konnten die deutschen Einheiten immer noch von ihren Vorteilen im Bereich der taktischen Führung und ihrer soliden Ausbildung im Gefecht der verbundenen Waffen profitieren. Diese Flexibilität erlaubte ihnen eine rasche Anpassung an das Kampfgelände Stadt.

Dieser Beitrag hat den Stadtkampf nur hinsichtlich der Operation »Barbarossa« untersucht und primär an einem Fallbeispiel. Trotzdem glaube ich damit aufgezeigt zu haben, dass der Kampf um Städte ein durchaus lohnendes Forschungsobjekt ist, das zu Unrecht bis heute ein Schattendasein fristete. Und zwar weit über die Operation »Barbarossa« hinaus, wie die Schlachten in Stalingrad, Arnhem, Aachen, Budapest und Berlin, um nur einige zu nennen, zeigen.

\begin{abstract}
This article examines the (German) conquest of cities during the German invasion of the Soviet Union, a theme that has been long neglected by military historians. The author shows that cities, due to the nature of Soviet infrastructure, became one of the main obstacles for German armored thrusts. Due to their logistical importance as the junctions for railroads and main roads, it was necessary for these urban areas to be seized; other logistical issues, however, such as a wasteful use of resources and high casualties, argued against capturing cities. The issue of taking cities also highlights the contradictory goals between Vernichtungskrieg and Blitzkrieg. An analysis of the tactical level and its attempt to cope with this new type of warfare is also included. Theses topics are considered in a case-study examination of the battle of Dnepropetrovsk (24.8.-30.9.1941).
\end{abstract}

